\title{
Avaliação de sistemas pró-degradantes na degradação termooxidativa do PEAD
}

\section{Evaluation of the pro-degradant systems in the thermooxidative degradation of HDPE}

\author{
Alex Sandro Babetto ${ }^{*}$, José Augusto Marcondes Agnelli e Sílvia Helena Prado Bettini ${ }^{1}$ \\ ${ }^{1}$ Laboratório de Polímeros, Departamento de Engenharia de Materiais - DEMa, Universidade Federal de \\ São Carlos - UFSCar, São Carlos, SP, Brasil \\ *alex.babetto@hotmail.com
}

\begin{abstract}
Resumo
Sistemas pró-degradantes fundamentados em estearatos de ferro e de manganês e o aditivo comercial d2w, fundamentado em sal orgânico de manganês, foram avaliados em filmes de polietileno de alta densidade (PEAD) em ambiente termooxidativo (estufa a $80{ }^{\circ} \mathrm{C}$ e ausência de luz). A degradação termooxidativa foi monitorada por propriedades mecânicas, espectroscopia de infravermelho e cromatografia por exclusão de tamanho. Os resultados mostram que ambos os metais aceleram a degradação termooxidativa do PEAD. Entretanto, o desempenho pró-degradante do estearato de manganês é significativamente superior ao do estearato de ferro. Para o ferro, a degradação aumenta com o aumento da concentração do metal, enquanto que o manganês apresenta valor máximo de degradação em função da concentração. $\mathrm{O}$ estado de oxidação do ferro não interfere no desempenho pró-degradante. $\mathrm{O}$ aditivo comercial $\mathrm{d} 2 \mathrm{w}^{\mathrm{TM}}$ acelera, significativamente, a termooxidação do PEAD, mas seu desempenho é inferior ao estearato de manganês provavelmente em função do conjunto de estabilizantes adicionados ao produto comercial.
\end{abstract}

Palavras-chave: degradação, PEAD, estearato de ferro, estearato de manganês, pró-degradante.

\begin{abstract}
The evaluation of pro-degradant systems based on iron and manganese in the form of metallic stearate, and $\mathrm{d} 2 \mathrm{w}^{\mathrm{TM}}$ commercial additive, based on organic salt of manganese, was carried out on films of high density polyethylene (HDPE) in thermal oxidative conditions $\left(80^{\circ} \mathrm{C}\right.$ oven and absence of light). The thermo oxidative degradation was monitored by mechanical properties, by infrared spectroscopy and by size exclusion cromatography. The results show that both metals accelerate thermal oxidative degradation of HDPE. However, the pro-degrading performance of manganese is significantly higher than iron. Considering iron, the degradation increases with increasing concentration of the metal, while manganese presents the maximum value of degradation depending on the concentration. The oxidation state of iron does not interfere with pro-degrading performance. The commercial additive $\mathrm{d} 2 \mathrm{w}^{\mathrm{TM}}$ significantly accelerates HDPE thermo-oxidation, but its performance is lower than manganese stearate as a function of the set of stabilizing additives to be added to the commercial product.
\end{abstract}

Keywords: degradation, HDPE, iron stearate, manganese stearate, pro-degradant.

\section{Introdução}

Quando os polímeros, entre eles os sintéticos, tornaram-se comercialmente importantes, surgiu a necessidade de se conhecer profundamente os processos de degradação dos mesmos e, com isto, prolongar a vida útil dos produtos fabricados a partir destes materiais. Atualmente, fundamentado no conceito de "produto ambientalmente correto", o mercado oferece algumas opções de aditivos, os prós-degradantes, que atuam no sentido contrário ao prolongamento da vida útil do produto, prometendo acelerar a degradação oxidativa do polímero e, desta forma, promover a biodegradação do mesmo. Os metais de transição, especialmente ferro $(\mathrm{Fe})$, manganês $(\mathrm{Mn})$ e cobalto (Co), são os principais constituintes dos aditivos pró-degradantes, os quais são denominados, comercialmente, de aditivos "oxi-bio". Aditivos pró-degradantes são comumente utilizados em produtos que possuam ciclo de vida útil relativamente curto e destino pós-consumo incerto $^{[1-11]}$.

Em função de ser referência como material para fabricação de produtos de ciclo de vida útil curto, como sacos e sacolas, por exemplo, o polietileno de alta densidade (PEAD) é um dos polímeros que mais recebe a adição dos aditivos pró-degradantes fundamentados em metais de transição. Por outro lado, os efeitos destes aditivos sobre o PEAD, ao contrário do polietileno de baixa densidade (PEBD) e 
polietileno linear de baixa densidade (PELBD), são ainda muito pouco conhecidos. Apesar de serem produzidos a partir do mesmo monômero, as diferenças nas condições de síntese conduzem a características distintas entre estas poliolefinas e, assim como o desempenho mecânico, o comportamento destes dois materiais são diferentes quando são submetidos a processos de degradação abiótico e/ou biótico ${ }^{[6,12,13]}$. Devido ao fato destes materiais serem utilizados em aplicações de uso único, seus prováveis destinos seriam os lixões, aterros e meio ambiente ${ }^{[2,3]}$. Neste caso, os aditivos pró-degradantes são adicionados ao PEAD para acelerar a degradação abiótica do mesmo e, com isto, transformar o PEAD em uma fonte de carbono para consumo microbiano, promovendo, assim, a biodegradação do produto. Para isto, o PEAD precisará receber grupos orgânicos oxigenados e sofrer fragmentação através de processos oxidativos de degradação, como a termooxidação, por exemplo. A efetiva assimilação do PEAD, pelos microrganismos, ocorrerá quando suas moléculas possuírem massas molares inferiores a 500 Dalton; para o PEAD, massas molares médias entre 2.000 e 3.000 Dalton possuem significativa fração de fragmentos com massa molar inferior a 500 Dalton $^{[10,11,14,15]}$. A participação dos metais de transição na degradação abiótica do PEAD, e de outras poliolefinas, ocorre na etapa de propagação do processo oxidativo de degradação; a aceleração da degradação é promovida através da decomposição dos hidroperóxidos pelos metais de transição, conforme as reações apresentadas na Figura $1^{[6,7,16]}$.

Os estudos realizados com agentes pró-degradantes, fundamentados em metal de transição, mostraram que estes agentes promovem a degradação oxidativa (termo e foto) do PEBD e PELBD, evidenciada, principalmente, através do aumento de grupos orgânicos funcionais oxigenados (cetonas, aldeídos, ésteres e ácidos carboxílicos). Os agentes são normalmente utilizados na forma de sais orgânicos, principalmente estearatos, e, além do Fe, Mn e Co, outros metais também são utilizados, como o cálcio, alumínio e titânio ${ }^{[17-38]}$.

Estudos demonstraram que a termooxidação e a fotooxidação do PEBD e PELBD aumentam em função do aumento da concentração do pró-degradante, fundamentado em metal de transição, na matriz polimérica ${ }^{[17,20,27,28,30,37]}$. Em processos termooxidativos, a degradação abiótica aumenta com o aumento da temperatura ${ }^{[1,21]}$ e com a pré-exposição das amostras à radiação UV e, quanto maior a pré-exposição, maior a termooxidação, mesmo na ausência da luz ${ }^{[29]}$. Por outro lado, a concentração de $\mathrm{O}_{2}$,

$$
\begin{aligned}
& \mathrm{ROOH}+\mathrm{M}^{\mathrm{n}+} \longrightarrow \mathrm{RO} \bullet+\mathrm{HO}^{-}+\mathrm{M}^{(\mathrm{n}+1)} \\
& \mathrm{ROOH}+\mathrm{M}^{(\mathrm{n}+1)} \longrightarrow \mathrm{ROO} \bullet+\mathrm{H}^{+}+\mathrm{M}^{\mathrm{n}+}
\end{aligned}
$$

\section{$2 \mathrm{ROOH} \longrightarrow \mathrm{RO} \bullet+\mathrm{ROO} \bullet+\mathrm{H}_{2} \mathrm{O}$ (c)}

Figura 1. Mecanismo de Haber-Weiss: decomposição do hidroperóxido por metal de transição $(\mathrm{R}=$ macromolécula de PEAD; $\mathrm{M}=$ metal de transição) $)^{[6,7,16]}$. no intervalo estudado (entre 5 e 20\%), não influencia no processo de termooxidação do PEBD e PELBD contendo agente pró-degradante ${ }^{[20]}$. Alguns estudos mostram que a presença de umidade intensifica a degradação abiótica oxidativa, mas o teor de umidade (intervalo estudado entre 60 e 100\% de umidade relativa) não interfere na intensidade da degradação ${ }^{[21,25]}$. Estudos realizados por Chiellini e colaboradores ${ }^{[23]}$ mostram que o tempo de indução para formação de carbonila, em algumas amostras, aumenta na presença de umidade, mas a intensidade de degradação abiótica oxidativa não é influenciada pela ausência ou presença de umidade. A umidade promove a hidrólise dos ésteres formados durante a degradação abiótica oxidativa, diminuindo a quantidade desta espécie na amostra ${ }^{[21]}$.

O principal mecanismo de ação do metal de transição é a decomposição dos hidroperóxidos e, com isto, ocorre aceleração do processo oxidativo de degradação; a transição entre os estados de oxidação do metal promove o mecanismo de decomposição ${ }^{[6,7,16]}$. A influência do estado de oxidação de alguns metais ( $\mathrm{Fe}, \mathrm{Mn}$ e $\mathrm{Co}$ ), relativo a termo e fotooxidação, foi avaliada e os resultados mostraram que o estado de oxidação não interfere, para a mesma concentração de estearato $(0,2$ e $0,5 \%)$, nos processos oxidativos de degradação do PEBD $^{[26,37]}$. Em processos de fotooxidação o desempenho dos metais, como pró-degradantes, é semelhante, mas na termooxidação a sequência de desempenho é $\mathrm{Co}>\mathrm{Mn}>\mathrm{Fe}^{[26]}$. Entretanto, estudos mostraram que o Co foi superior ao Fe em fotooxidação do PEBD e o Fe apresentou desempenho superior ao $\mathrm{Cu}$ neste mesmo estudo ${ }^{[37]}$. Estearato de $\mathrm{Fe}$ e estearato de $\mathrm{Ca}$ foram comparados, como pró-degradantes, em fotooxidação do PEBD e o $\mathrm{Fe}$ apresentou melhor desempenho, seja na fotooxidação natural ou acelerada ${ }^{[31]}$. Quanto aos tipos de polietilenos, a degradação abiótica oxidativa segue a seguinte ordem decrescente de intensidade: PEBD > PELBD > PEAD; a maior resistência aos processos oxidativos de degradação do PEAD é função da menor quantidade de carbono terciário nas moléculas e maior percentual de cristalinidade ${ }^{[30]}$. Agentes oxidantes como o permanganato de potássio, persulfato de potássio e peróxido de benzoíla aceleram os processos abióticos de degradação oxidativa no PEBD, principalmente a fotooxidação, uma vez que os grupos orgânicos funcionais oxigenados atuam como grupos cromóforos ${ }^{[39]}$.

Os estudos realizados sobre degradação abiótica e biodegradação de polietilenos mostram que a redução da massa molar e a introdução de oxigênio (aumento de até 5\% do peso) nas moléculas dos polietilenos estão associadas à etapa abiótica de degradação oxidativa e, os resultados da etapa abiótica, são fundamentais para o desenvolvimento da etapa biótica, na qual os microrganismos consomem os fragmentos oxigenados produzidos na etapa abiótica. Portanto, qualquer processo, produto ou procedimento que promova a degradação abiótica oxidativa dos polietilenos, também promoverá a degradação biótica deste polímero, desde que compostos de massa molar inferiores àqueles necessários para a assimilação do microrganismo sejam produzidos ${ }^{[19,20,23-25,30,34]}$. Por outro lado, o produto fabricado com o polímero, o qual se destina à biodegradação, precisa cumprir um período de utilização dentro de seu ciclo de vida e, para isto, deve garantir um tempo de vida útil antes que a degradação abiótica comprometa suas propriedades 
mecânicas e através da cinética da termooxidação é possível prever a durabilidade do produto, relativo a uma determinada propriedade mecânica, como, por exemplo, através do acompanhamento da \% de alongamento na ruptura ${ }^{[20,36]}$. O equilíbrio entre degradação abiótica oxidativa e desempenho mecânico durante a vida útil pode ser administrado através da quantidade e tipo de agente pró-degradante e/ou estabilizante utilizados na formulação do material polimérico ${ }^{[37,38]}$.

Este estudo teve por objetivo a avaliação de diferentes tipos de estearatos metálicos (MnII, FeII e FeIII), como aditivos pró-degradantes em $\mathrm{PEAD}$, quando submetidos à degradação termooxidativa a $80^{\circ} \mathrm{C}$. O trabalho apresenta o efeito do tipo de agente pró-degradante e da concentração dos mesmos na termooxidação de filmes de PEAD. Avaliou-se, também, a influência do estado de oxidação do Fe neste processo de degradação, bem como o desempenho de um aditivo comercial (AC). O monitoramento do ensaio de envelhecimento térmico foi realizado por espectroscopia no infravermelho (IR) e cromatografia por exclusão de tamanho (SEC).

\section{Materiais e Métodos}

\subsection{Materiais}

O PEAD utilizado no trabalho foi produzido pela empresa Braskem, sob o código GM9450F, com distribuição bimodal de massas molares, índice de fluidez (ASTM D1238) de $0.33 \mathrm{~g} / 10 \mathrm{~min}\left(190^{\circ} \mathrm{Ce} 5 \mathrm{~kg}\right)$ e $9,3 \mathrm{~g} / 10 \mathrm{~min}\left(190^{\circ} \mathrm{C} \mathrm{e} 21,6 \mathrm{~kg}\right)$ e densidade (ASTM D792) de $0,952 \mathrm{~g} / \mathrm{cm}^{3}$. Os aditivos pró-degradantes utilizados foram: estearato de manganês II (CAS 3353-05-7), fórmula molecular $\mathrm{C}_{36} \mathrm{H}_{70} \mathrm{O}_{4} \mathrm{Mn}$, massa molar igual a $621,89 \mathrm{~g} / \mathrm{mol}$, temperatura de fusão entre $100{ }^{\circ} \mathrm{C}$ e $110{ }^{\circ} \mathrm{C}$, pureza de $99,99 \%$, umidade máxima de $2 \%$, tamanho de partícula 99\%, no mínimo, em 200 mesh; estearato de ferro II (CAS 2980-59-8), fórmula molecular $\mathrm{C}_{36} \mathrm{H}_{70} \mathrm{O}_{4} \mathrm{Fe}$, massa molar igual a $622,81 \mathrm{~g} / \mathrm{mol}$, temperatura de fusão entre $78{ }^{\circ} \mathrm{Ce} 83^{\circ} \mathrm{C}$, pureza entre $95 \%$ e $100 \%$, umidade máxima de $3 \%$, tamanho de partícula $95 \%$, no mínimo, em 200 mesh; estearato de ferro III (CAS 555-36-2), fórmula molecular $\mathrm{C}_{54} \mathrm{H}_{105} \mathrm{O}_{6} \mathrm{Fe}$, massa molar igual a 906,25 g/mol, temperatura de fusão entre $90{ }^{\circ} \mathrm{Ce} 99^{\circ} \mathrm{C}$, pureza entre $95 \%$ e $100 \%$, tamanho de partícula $99 \%$, no mínimo, em 200 mesh. $\mathrm{O}$ aditivo pró-degradante comercial utilizado no trabalho foi fornecido pela Empresa RES Brasil Ltda (distribuidora oficial da Empresa Symphony Environmental Technologies plc, fabricante do aditivo) sob o código d2 $\mathrm{w}^{\mathrm{TM}}-93389$ (concentrado de sal orgânico de Manganês e antioxidantes em polietileno de baixa densidade).

\subsection{Métodos}

\subsubsection{Preparação das formulações e corpos de prova}

Antes do processo de extrusão, para preparação das formulações, o PEAD foi submetido ao processo de micronização no Micronizador Envitec ME-45 para aumentar a eficiência da mistura física entre o PEAD e os aditivos na forma de pó (estearato de ferro e de manganês); a mistura física foi realizada no misturador Henshell.

As formulações contendo PEAD micronizado e os agentes pró-degradantes estearato de manganês, estearato de ferro II e estearato de ferro III estão apresentadas na Tabela 1. A preparação foi conduzida em uma extrusora de rosca dupla co-rotante Process Equipment and Systems MP-2019 e a configuração das roscas está apresentada na Figura 2. As condições de processamento foram: perfil de temperatura igual a $170^{\circ} \mathrm{C}$ (alimentação), $175^{\circ} \mathrm{C}, 180^{\circ} \mathrm{C}$, $185^{\circ} \mathrm{C}$ e $195^{\circ} \mathrm{C}$ (cabeçote), rotação da rosca igual a $160 \mathrm{rpm}$ e vazão do alimentador igual a $1.068 \pm 22 \mathrm{~g} / \mathrm{hora}$; o tempo de residência médio do polímero neste processo de extrusão foi igual a $64 \pm 2$ segundos. A formulação d2w-34, em função de ser um concentrado do sal orgânico de Mn disperso em PEBD e por recomendação do fabricante, não foi submetida à preparação das formulações e o aditivo foi incorporado ao PEAD na mesma etapa de produção do filme e, com isto, foi submetida a apenas um processo de extrusão (produção do filme). A mistura física do AC com PEAD foi realizada com ambos os produtos na forma de grão.

A produção dos filmes, com espessura média de $47 \pm 6 \mu \mathrm{m}$ (ABNT NBR 14937) ${ }^{[40]}$, foi conduzida na extrusora de rosca dupla co-rotante, cuja configuração das roscas está apresentada na Figura 2, modelo MP-2019, marca Process

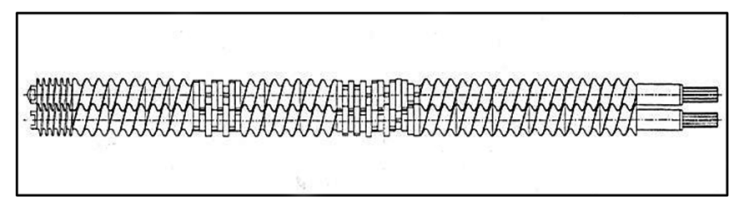

Figura 2. Configuração das roscas da extrusora rosca dupla co-rotante Process Equipment and Systems MP-2019.

Tabela 1. Quantidades dos metais de transição existentes nos filmes de PEAD.

\begin{tabular}{|c|c|c|c|}
\hline Formulação & $\begin{array}{c}\text { Fe } \\
\text { (mg/kg de PEAD) }\end{array}$ & $\begin{array}{c}\text { Mn } \\
\text { (mg/kg de PEAD) }\end{array}$ & $\begin{array}{c}d 2 w-34 \% \\
(\%)\end{array}$ \\
\hline Sem adição de pró-degradantes & $23 \pm 5$ & Não detectado & ----- \\
\hline Fe II-90 & $90 \pm 4$ & Não detectado & ---- \\
\hline Fe II-195 & $195 \pm 11$ & Não detectado & ----- \\
\hline Fe II-404 & $404 \pm 33$ & Não detectado & ----- \\
\hline Fe III-438 & $438 \pm 27$ & Não detectado & ----- \\
\hline Mn II-45 & $15 \pm 4$ & $45 \pm 7$ & ----- \\
\hline Mn II-191 & $18 \pm 2$ & $191 \pm 11$ & ------ \\
\hline Mn II-374 & $22 \pm 1$ & $374 \pm 31$ & ------ \\
\hline $\mathrm{d} 2 \mathrm{w}-34$ & $19 \pm 5$ & $34 \pm 4$ & 1,0 \\
\hline
\end{tabular}

(*) Percentual, em massa, do aditivo $\mathrm{d} 2 \mathrm{w}^{\mathrm{TM}}$ adicionado na formulação. 
Equipment and Systems em conjunto com a unidade de estiramento (puxador) marca AX Plásticos, modelo Chill Roll 16, e matriz de filme plano de $220 \mathrm{~mm}$ de largura marca AX Plásticos. As condições de processamento foram: perfil de temperatura igual a $170^{\circ} \mathrm{C}$ (alimentação), $175^{\circ} \mathrm{C}$, $180{ }^{\circ} \mathrm{C}, 185^{\circ} \mathrm{C}, 190{ }^{\circ} \mathrm{C}$ (cabeçote) e $195^{\circ} \mathrm{C}$ (matriz), rotação da rosca igual a $120 \mathrm{rpm}$, vazão do alimentador igual a $631 \pm 3 \mathrm{~g} /$ hora, velocidade linear do rolo puxador (velocidade de produção do filme) igual a $1.555 \mathrm{~mm} / \mathrm{min}$, velocidade linear do Rolo-1 igual a $1.555 \mathrm{~mm} / \mathrm{min}$ (Rolo-2 em trabalho livre), pressão do ar de resfriamento igual a $35 \mathrm{lb} / \mathrm{in}^{2}$ e temperatura do ar de resfriamento igual a $28^{\circ} \mathrm{C}$; o tempo de residência médio do polímero neste processo de extrusão foi igual a $179 \pm 4$ segundos. Os corpos de prova utilizados no monitoramento (ensaio mecânico, IR e SEC) foram extraídos a partir do filme extrudado utilizando-se o equipamento de corte marca Frank Prüfgeräte, modelo $\mathrm{GmbH}$. Os equipamentos utilizados no monitoramento são: (a) Maquina Universal de Ensaios, marca Instron, modelo 5569, distância inicial entre garras igual a $50 \mathrm{~mm}$ e velocidade do ensaio igual a $500 \mathrm{~mm} / \mathrm{min}$ (ASTM D882-12 e ASTM D3826-98), (b) Espectrofotômetro de Infravermelho marca Nicolet, modelo 6700 , resolução igual a $2 \mathrm{~cm}^{-1}$ e 64 varreduras (Índice de Carbonila) e (c) Cromatógrafo Viscotek, modelo GPC5351US - Viscotek Automated Conventional HT-GPC System (alteração da massa molar). A largura dos corpos de prova utilizados no ensaio mecânico (tração) foi de 25,4 mm (ASTM D882-12) e espessura de $47 \pm 6 \mu \mathrm{m}$.

A quantidade de metais de transição (Fe e Mn) existente nas formulações foi determinada nos filmes extrudados utilizando-se a técnica, não destrutiva, de Fluorescência de Raios-X, executada pelo equipamento marca HELMUT FISCHER $^{\circledR}$, modelo FICHERSCOPE ${ }^{\circledR}$ X-RAY XDV ${ }^{\circledR}$-SDD. Nas formulações 1, 5, 6, 7 e 9 não foi adicionado estearato de FeII, estearato de FeIII ou qualquer outra substância fundamentada em ferro. Entretanto, os resultados de Fluorescência de Raios-X mostram a existência de ferro nestas formulações, indicando que a incorporação do ferro nestas formulações pode ter ocorrido nas etapas de síntese e/ou processamentos do material, uma vez que a maioria dos equipamentos utilizados nestas etapas é fabricada com ligas metálicas fundamentadas em ferro.

\subsubsection{Degradação termooxidativa}

Amostras das formulações foram submetidas ao envelhecimento térmico em estufa a $80^{\circ} \mathrm{C}$ de temperatura e a degradação foi monitorada por espectroscopia de infravermelho através da determinação do índice de carbonila entre absorção a $1713 \mathrm{~cm}^{-1}$ (vibração $\mathrm{C}=\mathrm{O}$ ) e $730 \mathrm{~cm}^{-1}$ (vibração C-H); a escolha da referência em $730 \mathrm{~cm}^{-1}$ é função da estabilidade desta absorção. O equipamento utilizado foi o espectrofotômetro de infravermelho por transformada de Fourier Nicolet, modelo 6700, e as medidas foram conduzidas com resolução de $2 \mathrm{~cm}^{-1}$ e com 64 varreduras. O monitoramento foi realizado em triplicata, utilizando apenas um conjunto de corpo de prova por formulação (ensaio não destrutivo). Além do monitoramento por IR, foram realizados ensaios de SEC no tempo zero e no tempo final (4.545 horas) e o equipamento utilizado foi o cromatógrafo de permeação em gel de alta temperatura Viscotek, modelo GPC5351US - Viscotek Automated Conventional HT-GPC System, equipado com pré-coluna e três colunas Shodex HT-806M e detector de índice de refração (IR); os corpos de prova empregados no monitoramento por IR, foram utilizados para o ensaio de SEC no tempo final. Utilizou-se, para as análises de SEC, o solvente triclorobenzeno (TCB), e as condições de análise foram: temperatura de $150^{\circ} \mathrm{C}$ e vazão nas bombas principal e auxiliar de $1 \mathrm{ml} / \mathrm{min}$. O solvente foi pré-estabilizado com $1 \%$ de BHT.

\section{Resultados e Discussão}

As Figuras 3 a 5 apresentam os resultados de Índice de Carbonila (IC) determinados nas amostras de PEAD submetidas à termooxidação a $80^{\circ} \mathrm{C}$ durante 4.545 horas. Assim como para o PEBD e PELBD ${ }^{[17-38]}$, os resultados mostram que os agentes pró-degradantes fundamentados nos metais de transição Fe e Mn promovem a degradação termooxidativa do PEAD. Entretanto, a intensidade de aceleração do processo de termooxidação do Mn é significativamente superior ao

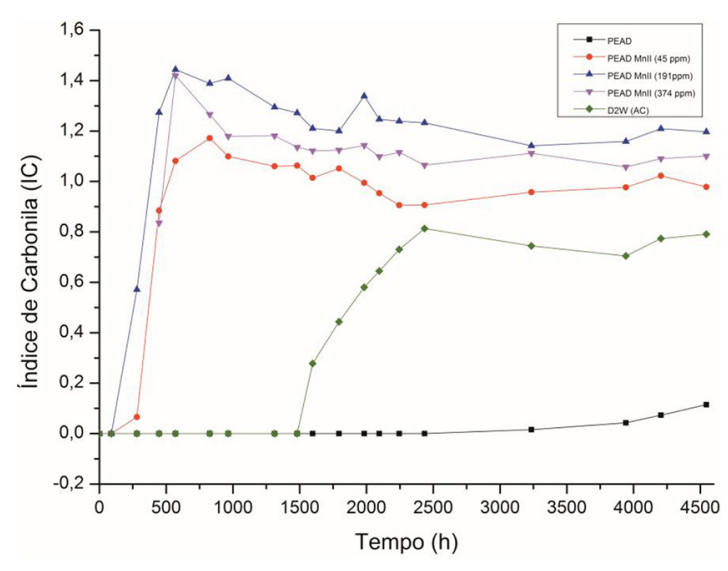

Figura 3. Degradação termooxidativa $\left(80^{\circ} \mathrm{C}\right)$, avaliada pelo índice de carbonila, do PEAD não aditivado com pró-degradante (PEAD) e aditivado com estearato de Mn, com quantidades variáveis de Mn.

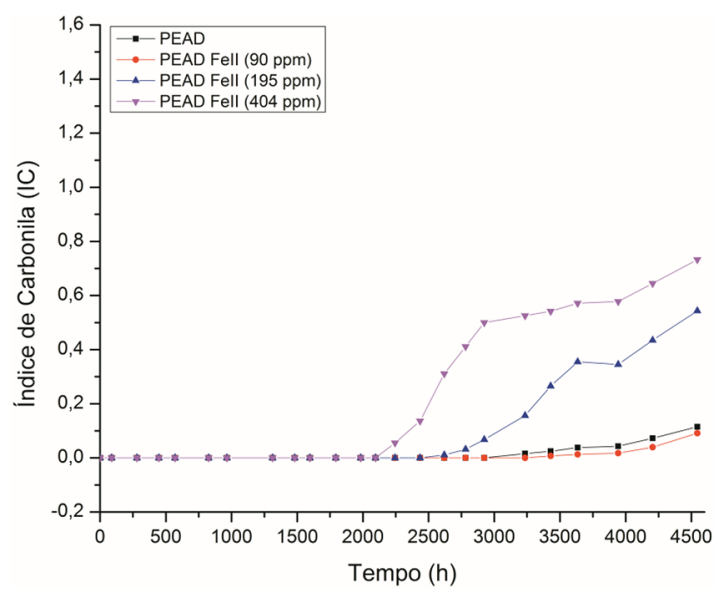

Figura 4. Degradação termooxidativa $\left(80^{\circ} \mathrm{C}\right)$, avaliada pelo índice de carbonila, do PEAD não aditivado com pró-degradante (PEAD) e aditivado com estearato de FeII, com quantidades variáveis de FeII. 


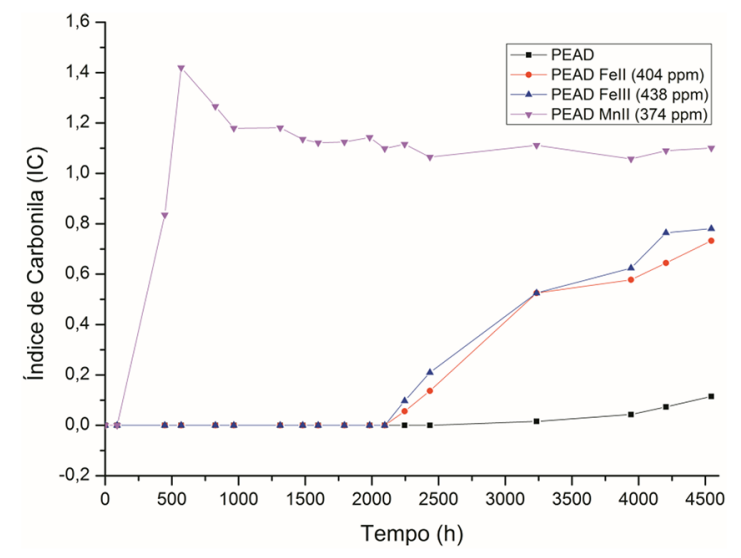

Figura 5. Degradação termooxidativa $\left(80^{\circ} \mathrm{C}\right)$, avaliada pelo índice de carbonila, do PEAD não aditivado com pró-degradante (PEAD) e aditivado com estearatos de FeII, FeIII ou MnII com quantidades aproximadas dos metais.

Fe, conforme apresentam os resultados; este comportamento é verificado em trabalhos anteriores ${ }^{[26,31,37]}$. Esta diferença ocorre para todas as formulações com concentração similar de metal de transição, como, por exemplo, entre as formulações FeII-195 (195 $\pm 11 \mathrm{mg} / \mathrm{kg})$ e MnII-191 (191 $\pm 11 \mathrm{mg} / \mathrm{kg})$, onde o tempo de indução, detectado pelo equipamento, para formação de carbonila na formulação FeII-195 é de, aproximadamente, 2.620 horas, enquanto que para a formulação MnII-191 o tempo de indução é inferior a 281 horas (mais de nove vezes menor); o tempo de indução para a formulação PEAD (formulação controle - sem adição de pró-degradante) é de, aproximadamente, 3.235 horas.

Além do tempo de indução para inicio da detecção do processo de termooxidativo, a cinética do processo também é diferente entre os metais, pois, conforme mostram os resultados, o Mn promove rápido aumento do IC nas três formulações que contém o metal na forma de estearato de $\mathrm{Mn}$, atingindo valores máximos de IC entre 570 horas (formulações MnII-191 e MnII-374) e 826 horas (formulação MnII-45) de exposição termooxidativa, enquanto que as formulações que contém estearato de FeII e estearato de FeIII (Figuras 3 a 5) apresentam pequeno aumento do IC e, ao final do ensaio (4.545 horas), ainda apresentavam tendência de crescimento do IC, mas um crescimento lento. É importante destacar que a formulação FeII-90 $(90 \pm 4 \mathrm{mg} / \mathrm{kg})$ apresenta comportamento semelhante à formulação de PEAD não aditivado com pró-degradante (controle) e este resultado indica que concentrações iguais ou inferiores a $90 \mathrm{mg} / \mathrm{kg}$ não influenciam, até o tempo avaliado, no processo termooxidativo, a $80^{\circ} \mathrm{C}$, de degradação do PEAD. Quanto à influência da concentração de Fe no PEAD (Figura 4), a degradação termooxidativa aumenta com o aumento da concentração de Fe, conforme observado para o PEBD e $\operatorname{PELBD}^{[17,20,27,28,30,37]}$.

Os resultados (Figura 5) também mostram que o estado de oxidação do Fe (formulações FeII-404 e FeIII438) não interfere no processo de termooxidação do PEAD a $80^{\circ} \mathrm{C}$, indicando que as reações (a) e (b) da Figura 1 ocorrem com a mesma intensidade, resultado contrário ao que foi observado em trabalhos anteriores para o $\mathrm{PEBD}^{[26,37]}$, uma vez que em concentrações diferentes de FeII e FeIII (mesma concentração em estearato de ferro significa, aproximadamente, $45 \%$ menos de ferro para a formulação que contém o estearato de FeIII) o PEBD não apresentou diferença no processo termooxidativo. É importante destacar que na comparação entre trabalhos, anteriores e este, os polímeros são diferentes (PEBD e PEAD, respectivamente), mas esperava-se que o FeIII promovesse maior termooxidação ao PEAD que o FeII quando avaliados na mesma concentração do metal. Uma provável hipótese para este comportamento pode ser devido ao discreto efeito acelerador da termooxidação do ferro e, por isto, diferenças superiores a $45 \%$ entre as concentrações do FeII e FeIII ou tempos de ensaio significativamente maiores poderão destacar o efeito promotor da termooxidação a $80^{\circ} \mathrm{C}$ do FeIII relativo ao FeII no PEAD no intervalo de tempo estudado. É importante destacar que a temperatura de termooxidação está compreendida no intervalo de temperatura de fusão do estearato de FeII $\left(78-83^{\circ} \mathrm{C}\right)$ e é possível que o estado físico (líquido) deste agente pró-degradante tenha interferido na ação pró-degradante ao PEAD quando comparado aos estearatos de FeIII e de MnII, os quais apresentam intervalo de temperatura de fusão $\left(90-99^{\circ} \mathrm{C}\right.$ e $100-110^{\circ} \mathrm{C}$, respectivamente) superior à temperatura de termooxidação. Entretanto, para se concluir sobre a influência do estado físico do estearato de FeII na ação pró-degrante do agente ao PEAD serão realizados novos estudos.

Como foi destacado anteriormente, o Mn é, como agente acelerador, significativamente superior ao $\mathrm{Fe}$ no processo de termooxidação, a $80^{\circ} \mathrm{C}$, do PEAD (Figura 5). Entretanto, ao contrário do $\mathrm{Fe}$ e de estudos realizados anteriormente com PEBD aditivado com $\mathrm{Mn}^{[17,20,27,28,30,37]}$, a termooxidação do PEAD em função da concentração de Mn (na forma de estearato de manganês) apresenta, no intervalo de concentração estudado, intensidade máxima com a formulação intermediária MnII- 191 (Figura 3). Apesar de discreta, a superioridade do IC da formulação com concentração intermediária induz à interpretação de que existe uma quantidade ótima de $\mathrm{Mn}$, acima ou abaixo da qual o desempenho do metal como agente promotor da termooxidação do PEAD é inferior. Não podemos deixar de associar o desempenho, na termooxidação a $80^{\circ} \mathrm{C}$, das formulações MnII-45 e MnII-191, comparadas à formulação MnII-374, com a degradação termo-mecânica ocorrida nos processos de extrusão, conforme mostram os resultados (Tabelas 2 e 3) de SEC e ensaio mecânico de tração no tempo zero; relativo às propriedades mecânicas, os efeitos da degradação nos processos de extrusão podem ser nitidamente observados nas propriedades de tensão na ruptura e deformação na ruptura. É possível observar que, durante o processo de extrusão, a degradação é inversamente proporcional à concentração do Mn e estes resultados podem explicar o elevado efeito acelerador da termooxidação do PEAD em estufa a $80^{\circ} \mathrm{C}$ para as formulações MnII-45 e MnII-191 comparadas à MnII-374, uma vez que o PEAD das formulações MnII-45 e MnII-191 iniciam o processo de termooxidação em estufa significativamente mais degradadas que a formulação MnII-374. Os resultados (Tabelas 2 e 3 e Figura 3) também indicam que o efeito de proporcionalidade inversa entre a concentração de Mn e a degradação termo-mecânica pode ter ocorrido apenas nos processos de extrusão, enquanto que na termooxidação em 
Tabela 2. Massa molar em função do tempo de termooxidação $\left(80^{\circ} \mathrm{C}\right)$ do PEAD.

\begin{tabular}{|c|c|c|c|c|}
\hline \multirow[t]{2}{*}{ Formulação } & Mn (Dalton) & Mw (Dalton) & Mn (Dalton) & Mw (Dalton) \\
\hline & Zero h ${ }^{(*)}$ & Zero $\mathbf{h}^{(*)}$ & $4545 \mathrm{~h}$ & $4545 \mathrm{~h}$ \\
\hline Sem adição de pró-degradante & 36.583 & 649.395 & Inconclusivo & Inconclusivo \\
\hline FeII-90 & 34.908 & 560.723 & 5.265 & 288.122 \\
\hline FeII-190 & 32.173 & 593.644 & 4.580 & 67.803 \\
\hline FeII-404 & 28.485 & 580.004 & 3.583 & 29.995 \\
\hline FeIII-438 & 26.835 & 557.807 & 3.349 & 26.636 \\
\hline MnII-45 & 5.665 & 477.604 & 2.656 & 31224 \\
\hline MnII-191 & 11.797 & 509.748 & 2.001 & 16.493 \\
\hline MnII-374 & 18.036 & 593.153 & 232 & 12.637 \\
\hline $\mathrm{d} 2 \mathrm{w}-34$ & 35.171 & 619.386 & 241 & 25.900 \\
\hline
\end{tabular}

$(*)$ Zero hora - SEC realizado após 24 horas $\left(23^{\circ} \mathrm{C}\right.$ - isento de luz) da extrusão dos filmes.

Tabela 3. Propriedades mecânicas* após 24 horas da produção dos filmes $\left(23^{\circ} \mathrm{C}\right.$ e ausência de luz).

\begin{tabular}{lccccccccc}
\hline \multicolumn{1}{c}{ Propriedades } & PEAD & FeII & FeII & FeII & FeIII & MnII & MnII & MnII & d2w \\
& & $\mathbf{9 0}$ & $\mathbf{1 9 0}$ & $\mathbf{4 0 4}$ & $\mathbf{4 3 8}$ & $\mathbf{4 5}$ & $\mathbf{1 9 1}$ & $\mathbf{3 7 4}$ & $\mathbf{3 4}$ \\
\hline Tensão de escoamento & $25,2 \pm 2,9$ & $28,2 \pm 2,6$ & $26,8 \pm 2,0$ & $26,7 \pm 3,6$ & $25,6 \pm 3,2$ & $29,8 \pm 1,8$ & $28,9 \pm 2,2$ & $26,4 \pm 2,4$ & $22,5 \pm 1,6$ \\
(MPa) & & & & & & & & & \\
Resistência na ruptura & $55,8 \pm 10,3$ & $62,8 \pm 8,5$ & $58,1 \pm 7,2$ & $57,0 \pm 10,8$ & $63,2 \pm 12,6$ & $30,2 \pm 1,8$ & $29,7 \pm 3,0$ & $47,4 \pm 4,2$ & $65,7 \pm 6,1$ \\
(MPa) & & & & & & & & & \\
$\begin{array}{l}\text { Deformação na ruptura } \\
\text { (\%) }\end{array}$ & $470 \pm 48$ & $472 \pm 56$ & $463 \pm 51$ & $480 \pm 94$ & $463 \pm 55$ & $69 \pm 64$ & $104 \pm 82$ & $490 \pm 84$ & $499 \pm 41$ \\
\hline
\end{tabular}

(*) ASTM D882-12 - velocidade do ensaio igual a $500 \mathrm{~mm} / \mathrm{min}$, distância entre garras igual a $50 \mathrm{~mm}$, largura do corpo de prova igual a $25,4 \mathrm{~mm}$ e espessura média igual a $47 \pm 6 \mu \mathrm{m}$. (**) PEAD não aditivado com pró-degradante.

estufa a $80{ }^{\circ} \mathrm{C}$ ocorre o efeito de proporcionalidade direta e esta ocorrência conduz à equiparação nos valores de IC ao longo do ensaio na estufa a $80^{\circ} \mathrm{C}$ de temperatura e os resultados de SEC (Tabela 2) reforçam este comportamento. A formulação MnII-374, mesmo apresentando tempo de indução do IC maior, o ponto de máximo ocorre no mesmo tempo de envelhecimento acelerado da formulação MnII-191 e antes da formulação MnII-45 e, no tempo final de ensaio (4.545h) o Mw e, principalmente, o Mn da formulação MnII-374 são os menores valores entre todas as formulações estudas, inclusive quando comparado à formulação MnII-191, que apresentou o maior valor máximo de IC e menor tempo de indução do IC. O comportamento de proporcionalidade inversa, durante o processo de extrusão, pode ser explicado pelos fenômenos: (a) o aumento da concentração de estearato de Mn pode promover a interação entre as moléculas de estearato de $\mathrm{Mn}$, gerando aglomerados das mesmas e, com isto, inibindo a ação como pró-degradante ao PEAD e/ou (b) o efeito lubrificante do estearato reduz o cisalhamento entre as moléculas de PEAD e entre as moléculas de PEAD e as partes metálicas da extrusora (rosca, cilindro e matriz), minimizando a degradação térmica e mecânica durante o processamento. O fenômeno (a) é pouco provável, pois caso os aglomerados de estearato de $\mathrm{Mn}$ se formassem durante o processo de extrusão, provavelmente permaneceriam quando o PEAD se solidifica (grão e filme) e, com isto, esperava-se o mesmo comportamento de proporcionalidade inversa na termooxidação a $80^{\circ} \mathrm{C}$, o que, conforme resultados, não ocorreu. Entretanto, os dois fenômenos devem ser investigados com maior profundidade. Por outro lado, a proporcionalidade direta em estufa pode ser função da maior concentração do $\mathrm{Mn}$ no PEAD, indicando que a termooxidação aumenta com o aumento da concentração do $\mathrm{Mn}$, assim como ocorre com o Fe.

Os resultados de IR mostram que o Mn acelera significativamente o processo termooxidativo do PEAD a $80{ }^{\circ} \mathrm{C}$, o qual atinge valores máximos de IC em tempos inferiores, quando comparado ao PEAD Puro. Após atingir o valor máximo, o IC apresenta uma leve queda, mas mantém valores elevados de IC. As principais alterações, relativas ao tempo zero, no espectro de IR do PEAD degradado estão localizadas entre 1050 e $1450 \mathrm{~cm}^{-1}$ e entre 1600 e $1800 \mathrm{~cm}^{-1}$, conforme pode ser observado na Figura 6. Estes intervalos correspondem a diversos grupos orgânicos oxigenados, principalmente cetonas (1712-1723), ácido carboxílico (1250 e 1680-1725), ésteres (1050-1300), aldeídos (1675-1730), lactonas e perácidos (1770-1780) e éteres (1060-1300), os quais são produtos do processo de degradação oxidativa do $\operatorname{PEAD}^{[21,23,41-43]}$.

O intervalo entre 1300 e $1450 \mathrm{~cm}^{-1}$ pode ser devido à flexão das ligações carbono e hidrogênio de diferentes espécies de hidrocarbonetos como grupos metila, metileno, isopropila entre outros, e o intervalo entre 850 e $1000 \mathrm{~cm}^{-1}$ pede ser relacionado à formação de insaturação; estas espécies podem ser formadas durante o processo de degradação, principalmente em função da cisão da cadeia polimérica ${ }^{[11,42]}$. Os pontos de máximo e a relativa estabilização do IC observados, principalmente, nas formulações contendo $\mathrm{Mn}$ não significa que o PEAD recuperou sua estrutura química ou que o processo de degradação não ocorre mais. O que pode ter ocorrido, conforme mostra a Figura 6, é que os 


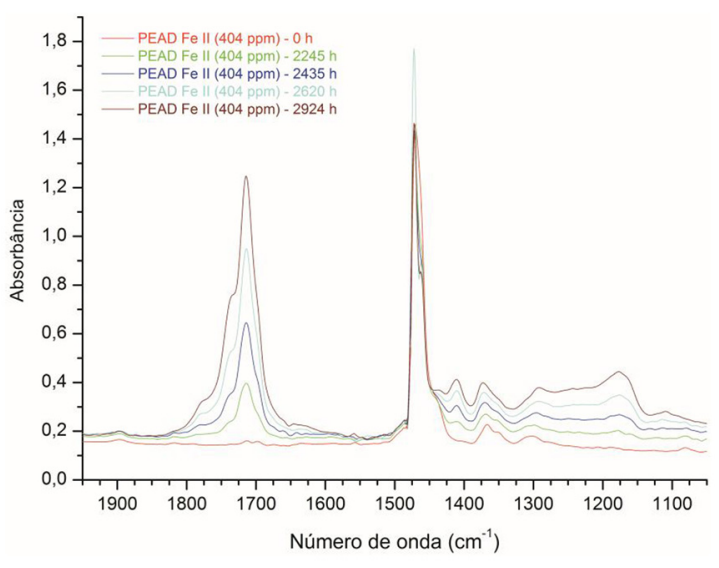

Figura 6. Variação dos espectros de IR em função do tempo de degradação termooxidativa a $80{ }^{\circ} \mathrm{C}$ para a formulação FeII-404.

grupos carbonílicos responsáveis pela absorção de radiação infravermelho na região de $1713 \mathrm{~cm}^{-1}$ experimentaram, ao longo do processo de degradação, alterações na cadeia carbônica principal e no próprio grupo funcional e, com isto, ocorreram deslocamentos da região de absorção em detrimento do aumento da absorção na região de $1713 \mathrm{~cm}^{-1}$. Não observamos este comportamento nas formulações contendo $\mathrm{Fe}$ (FeII e FeIII), mas isto não significa que ele não possa ocorrer, visto que as formulações contendo Fe apresentavam, ao final do ensaio, tendência de crescimento do IC e, sendo assim, o comportamento observado para o $\mathrm{Mn}$ pode ocorrer com o Fe em tempos mais elevados de ensaio.

$\mathrm{O}$ aditivo comercial $\left(\mathrm{d} 2 \mathrm{w}^{\mathrm{TM}}\right)$, fundamentado em sal orgânico de manganês, apresentou comportamento intermediário entre a formulação que contém maior concentração de $\mathrm{Fe}$ (FeII-404 e FeIII-438) e a formulação que contém a menor concentração de Mn (Figura 3). A concentração do Mn na formulação d2w-34 (34 $\pm 4 \mathrm{mg} / \mathrm{kg})$ é, considerando o desvio padrão, igual à formulação MnII-45 $(45 \pm 7 \mathrm{mg} / \mathrm{kg})$, mas o tempo de indução para início da termooxidação ao PEAD da formulação contendo $\mathrm{d} 2 \mathrm{w}$-34 é significativamente superior e um dos motivos deste comportamento é relativo à diferença de degradação no tempo zero entre as formulações (Tabelas 2 e 3 ). Entretanto, após o tempo de indução as acelerações das reações de termooxidação do d2w-34 e MnII-45 são semelhantes. A distinção entre os tempos de indução para as formulações d2w-34 e MnII-45 pode ser explicada por dois fatores: (a) o PEAD da formulação $\mathrm{d} 2 \mathrm{w}^{\mathrm{TM}}$ foi submetido apenas ao processo de extrusão do filme (menor exposição térmica e mecânica), pois o aditivo d2w-34 é fornecido na forma de concentrado disperso em PEBD e, conforme recomendação do fabricante, não é necessário realizar a pré-incorporação do mesmo, e (b) conforme informações do fabricante, o aditivo concentrado possui, além do sal orgânico de Mn, um conjunto de estabilizantes (identificação não informada) com o objetivo de, conforme condições de processamento e estocagem específicas, controlar a vida útil do produto fabricado com o aditivo d2w-34. Os resultados também mostram que o d2w-34 apresenta, comparado à formulação PEAD Puro (controle), significativa redução do tempo de indução e maior aceleração da termooxidação ao PEAD, evidenciando intenso processo de degradação, inclusive na comparação dos valores de IC no tempo final de ensaio.

\section{Conclusões}

Em função dos resultados, conclui-se que os metais de transição Fe e Mn aceleram a degradação térmica do PEAD em processos de extrusão e em estufa a $80^{\circ} \mathrm{C}$, sendo o $\mathrm{Mn}$ significativamente mais eficiente na redução do tempo de indução e na aceleração do processo. O Fe e o Mn apresentam comportamentos distintos quanto à influência da concentração do metal, enquanto o $\mathrm{Fe}$ apresenta proporcionalidade direta, o Mn mostra, para o intervalo de concentração estudado, comportamento de máximo em função da concentração do Mn; este comportamento pode estar associado à influência do Mn na degradação do PEAD na etapa de processamento, como indicam os resultados de ensaios mecânicos e SEC. O estado de oxidação do Fe não influencia, para a concentração avaliada, o processo de termooxidação do PEAD em estufa a $80^{\circ} \mathrm{C}$. Após o tempo de indução, o qual é superior à formulação MnII-45 (concentrações, aproximadamente, iguais de $\mathrm{Mn}$ ), a formulação contendo aditivo $\mathrm{d} 2 \mathrm{w}^{\mathrm{TM}}$ acelera significativamente o processo de termooxidação do PEAD quando exposto em estufa a $80{ }^{\circ} \mathrm{C}$.

\section{Agradecimentos}

Os autores agradecem à CAPES pela concessão de bolsa de doutorado de Alex Sandro Babetto, à empresa Symphony Environmental Technologies plc e à empresa RES Brasil Ltda pela doação do aditivo comercial d2 $\mathrm{w}^{\mathrm{TM}}$, à empresa Dufaplast Indústria de Plásticos - EIRELI EPP pela micronização do PEAD e à empresa HELMUT FISCHER $^{\circledR}$ pelas análises de Fluorescência de Raios-X para a quantificação dos metais.

\section{Referências}

1. Uda, M. J. (2011). Logística reversa da reciclagem de garrafas PET em Curitiba (Dissertação de mestrado). Instituto de Tecnologia para o Desenvolvimento (LACTEC), Curitiba.

2. Fabro, A. T., Lindemann, C., \& Vieira, S. C. (2007). Utilização de sacolas plásticas em supermercados. Revista Ciências do Ambiente, 3(1), 15-23.

3. Tonello, D., Guissoni, L. S., Rizzo, M. R., Ribeiro, S. P., \& Tisott, S. T. (2011). A polêmica da redução e extinção do uso das sacolas plásticas nos supermercados. Fórum Ambiental da Alta Paulista, 7(4), 709-725. http://dx.doi. org/10.17271/19800827742011146.

4. Vieira, S., \& Carrillo, M. (2009). Sacolinha plástica: preocupação ambiental ou disputa por um mercado. Revista Reciclagem Moderna, 17, p. 24-31.

5. Vieira, S. (2011). Sacolas plásticas: os impactos no setor de reciclagem com o fim da sacola plástica. Revista Reciclagem Moderna, 27, p. 24-29.

6. De Paoli, M. A. (2008). Degradação e estabilização de polímeros. São Paulo: Artliber.

7. Ammala, A., Bateman, S., Dean, K., Petinakis, E., Sangwan, P., Wong, S., Yuan, Q., Yu, L., Patrick, C., \& Leong, K. H. (2011). An overview of degradable and biodegradable polyolefins. Progress in Polymer Science, 36(8), 1015-1049. http://dx.doi. org/10.1016/j.progpolymsci.2010.12.002. 
8. Koutny, M., Lemaire, J., \& Delort, A.-M. (2006). Biodegradation of polyethylene films with prooxidant additives. Chemosphere, 64(8), 1243-1252. http://dx.doi.org/10.1016/j.chemosphere.2005.12.060. PMid:16487569.

9. Franchetti, S. M. M., \& Marconato, J. C. (2006). Polímeros biodegradáveis: uma solução parcial para diminuir a quantidade dos resíduos plásticos. Química Nova, 29(4), 811-816.

10. Luckachan, G. E., \& Pillai, C. K. S. (2011). Biodegradable polymers: a review on recent trends and emerging perspectives. Journal of Polymers and the Environment, 19(3), 637-676. http://dx.doi.org/10.1007/s10924-011-0317-1.

11. Lucas, N., Bienaime, C., Belloy, C., Queneudec, M., Silvestre, F., \& Nava-Saucedo, J.-E. (2008). Polymer biodegradation: mechanisms and estimation techniques. Chemosphere, 73(4), 429-442. http://dx.doi.org/10.1016/j.chemosphere.2008.06.064. PMid:18723204.

12. Malpass, D. B. (2010). Introduction to industrial polyethylene. Hoboken: John Wiley \& Sons.

13. Coutinho, F. M. B. (2003). Polietileno: principais tipos, propriedades e aplicações. Polímeros: Ciência e Tecnologia, 13(1), 1-13. http://dx.doi.org/10.1590/S0104-14282003000100005

14. Lehninger, A. L. (1987) Biquímica (v. 2). São Paulo: Editora Edgard Blücher Ltda.

15. Albertsson, A.-C., Andersson, S. O., \& Karlsson, S. (1987). The mechanism of biodegradation of polyethylene. Polymer Degradation \& Stability, 18(1), 73-87. http://dx.doi. org/10.1016/0141-3910(87)90084-X.

16. Osawa, Z. (1988). Role of metals and metal-deactivators in polymer degardation. Polymer Degradation \& Stability, 20(3-4), 203-236. http://dx.doi.org/10.1016/0141-3910(88)90070-5.

17. Albertsson, A.-C., Erlandsson, B., Hakkarainen, M., \& Karlsson, S. (1998). Molecular weight changes and polymeric matrix changes correlated with the formation of degradation products in biodegraded polyethylene. Journal of Environmental Polymer Degradation, 6(4), 187-195. http:// dx.doi.org/10.1023/A:1021873631162.

18. Chiellini, E., Corti, A., \& Swift, G. (2003). Biodegradation of thermally-oxidized, fragmented low-density polyethylenes. Polymer Degradation \& Stability, 81(2), 341-351. http://dx.doi. org/10.1016/S0141-3910(03)00105-8.

19. Bonhomme, S., Cuer, A., Delort, A.-M., Lemaire, J., Sancelme, M., \& Scott, G. (2003). Environmental biodegradation of polyethylene. Polymer Degradation \& Stability, 81(3), 441452. http://dx.doi.org/10.1016/S0141-3910(03)00129-0.

20. Jakubowicz, I. (2003). Evaluation of degradability of biodegradable polyethylene (PE). Polymer Degradation \& Stability, 80(1), 39-43. http://dx.doi.org/10.1016/S0141-3910(02)00380-4.

21. Jakubowicz, I., Yarahmadi, N., \& Petersen, H. (2006). Evaluation of the rate of abiotic degradation of biodegradable polyethylene in various environments. Polymer Degradation \& Stability, 91(7), 1556-1562. http://dx.doi.org/10.1016/j. polymdegradstab.2005.09.018

22. Sheikh, N., Akhavan, A., Naimian, F., Khoylou, F., Hassanpour, S., \& Sohrabpour, M. (2006). Formulation of a photosensitized polyethylene film; its structure and property variation under the weathering conditions of tehran. Journal of Polymers and the Environment, 14(1), 103-109. http://dx.doi.org/10.1007/ s10924-005-8712-0.

23. Chiellini, E., Corti, A., D’Antone, S., \& Baciu, R. (2006). Oxo-biodegradable carbon backbone polymers: oxidative degradation of polyethylene under accelerated test conditions. Polymer Degradation \& Stability, 91(11), 2739-2747. http:// dx.doi.org/10.1016/j.polymdegradstab.2006.03.022.

24. Chiellini, E., Corti, A., \& D'Antone, S. (2007). Oxo-biodegradable full carbon backbone polymers: biodegradation behaviour of thermally oxidized polyethylene in an aqueous medium.
Polymer Degradation \& Stability, 92(7), 1378-1383. http:// dx.doi.org/10.1016/j.polymdegradstab.2007.03.007.

25. Ojeda, T. F. M., Dalmolin, E., Forte, M. M. C., Jacques, R. J. S., Bento, F. M., \& Camargo, F. A. O. (2009). Abiotic and biotic degradation of oxo-biodegradable polyethylenes. Polymer Degradation \& Stability, 94(6), 965-970. http://dx.doi. org/10.1016/j.polymdegradstab.2009.03.011.

26. Roy, P. K., Surekha, P., Raman, R., \& Rajagopal, C. (2009). Investigating the role of metal oxidation state on the degradation behaviour of LDPE. Polymer Degradation \& Stability, 94(7), 1033-1039. http://dx.doi.org/10.1016/j. polymdegradstab.2009.04.025.

27. Roy, P. K., Singh, P., Kumar, D., \& Rajagopal, C. (2010). Manganese Stearate Initiated Photo-Oxidative and ThermoOxidative Degradation of LDPE, LLDPE and Their Blends. Journal of Applied Polymer Science, 117, 524-533. http:// dx.doi.org/10.1002/app.31252.

28. Magagula, B., Nhlapo, N., \& Focke, W. W. (2009). Mn2A1LDH- and Co2Al-LDH stearate as photodegradants for LDPE film. Polymer Degradation \& Stability, 94(6), 947-954. http:// dx.doi.org/10.1016/j.polymdegradstab.2009.03.007.

29. Vogt, N. B., \& Kleppe, E. A. (2009). Oxo-biodegradable polyolefins show continued and increased thermal oxidative degradation after exposure to light. Polymer Degradation \& Stability, 94(4), 659-663. http://dx.doi.org/10.1016/j. polymdegradstab.2009.01.002.

30. Fontanella, S., Bonhomme, S., Koutny, M., Husarova, L., Brusson, J.-M., Courdavault, J.-P., Pitteri, S., Samuel, G., Pichon, G., Lemaire, J., \& Delort, A. M. (2010). Comparison of the biodegradability of various polyethylene films containing pro-oxidant additives. Polymer Degradation \& Stability, 95(6), 1011-1021. http://dx.doi.org/10.1016/j. polymdegradstab.2010.03.009.

31. Pablos, J. L., Abrusci, C. I., Marin, I., López-Marín, J., Catalina, F., Espí, E., \& Corrales, T. (2010). Photodegradation of polyethylenes: comparative effect of $\mathrm{Fe}$ and $\mathrm{Ca}$-stearates as pro-oxidant additives. Polymer Degradation \& Stability, 95(10), 2057-2064. http://dx.doi.org/10.1016/j.polymdegradstab.2010.07.003.

32. Manangan, T., Shawaphun, S., Sangsansiri, D., Changcharoen, J., \& Wacharawichanant, S. (2010). Nano-sized titanium dioxides as photo-catalysts in degradation of polyethylene and polypropylene packagings. Science Journal Ubon Ratchathani University, 1(2), 14-20.

33. Yang, R., Christensen, P. A., Egerton, T. A., White, J. R., \& Maltby, A. (2010). Spectroscopic Studies of photodegradation of polyethylene films containing $\mathrm{TiO} 2$ nanoparticles. Journal of Applied Polymer Science, 119(3), 1330-1338. http://dx.doi. org/10.1002/app.31669.

34. Abrusci, C., Pablos, J. L., Corrales, T., López-Marín, J., Marín, I., \& Catalina, F. (2011). Biodegradation of photodegraded mulching films based on polyethylenes and stearates of calcium and iron as pro-oxidant additives. International Biodeterioration \& Biodegradation, 65(3), 451-459. http://dx.doi.org/10.1016/j. ibiod.2010.10.012.

35. Konduri, M. K. R., Koteswarareddy, G., Kumar, D. B. R., Reddy, B. V., \& Narasu, M. L. (2011). Effect of pro-oxidants on biodegradation of polyethylene (LDPE) by indigenous fungal isolate, aspergillus oryzae. Journal of Applied Polymer Science, 120(6), 3536-3545. http://dx.doi.org/10.1002/app.33517.

36. Jakubowicz, I., Yarahmadi, N., \& Arthurson, V. (2011). Kinetics of abiotic and biotic degradability of low-density polyethylene containing prodegradant additives and its effect on the growth of microbial communities. Polymer Degradation \& Stability, 96(5), 919-928. http://dx.doi.org/10.1016/j. polymdegradstab.2011.01.031. 
37. Focke, W. W., Mashele, R. P., \& Nhlapo, N. S. (2011). Stabilization of low-density polyethylene films containing metal stearates as photodegradants. Journal of Vinyl \& Additive Technology, 17(1), 21-27. http://dx.doi.org/10.1002/ vnl.20248.

38. Jakubowicz, I., \& Enebro, J. (2012). Effects of reprocessing of oxobiodegradable and non-degradable polyethylene on the durability of recycled materials. Polymer Degradation \& Stability, 97(3), 316-321. http://dx.doi.org/10.1016/j. polymdegradstab.2011.12.011.

39. Roy, P. K., Surekha, P., \& Rajagopal, C. (2011). Surface oxidation of low-density polyethylene films to improve their susceptibility toward environmental degradation. Journal of Applied Polymer Science, 122(4), 2765-2773. http://dx.doi. org/10.1002/app.34097.
40. Associação Brasileira de Normas Técnicas - ABNT. (2010). NBR 14937: sacolas plásticas tipo camiseta: requisitos e métodos de ensaio. Rio de Janeiro: ABNT.

41. Boyd, R. N., \& Morrison, R. T. (1983). Química Orgânica. Lisboa: Fundação Calouste Gulbenkian.

42. Agnelli, J. A. M. (2007). Degradação e estabilização de polímeros (Apostila do Núcleo de Reologia e Processamento de Polímeros). São Carlos: Universidade Federal de São Carlos.

43. Gugumus, F. (2001). Re-examination of the thermal oxidation reactions of polymers 1: new views of an old reaction. Polymer Degradation and Stability, 74, 327-339.

Enviado: Fev. 13, 2015 Revisado: Maio 25, 2015 Aceito: Jun. 01, 2015 